\title{
SOME ASPECTS OF TEXTURE IN FERROFLUIDS
}

\section{R.W. Chantre11}

School of Physics and Astronomy, Lancashire Polytechnic, Preston, Lancs., PR1 2TQ, U.K.

Résumé - Les propriétês des ferrofluides sont affectées jusqu'à un certain point par deux types distincts de texture. Le premier est une texture "d'orientation" caractêrisé par l'orientation des particules dans un champ magnétique. Il se manifeste lorsque le couplage entre le moment magnétique et les axes cristallographiques de la particule est fortement anisotrope. Un second type de texture résulte de 1 'interaction magnétique dipolaire entre les particules, qui peut induire une texture "spatiale". Ce papier décrit les effets de chaque type de texture sur les propriêtés des ferrofluides.

\begin{abstract}
The properties of ferrofluids are affected to some degree by two distinct types of texture. The first is an 'orientational' texture, characterised by the orientation of particles by a magnetic field. This arises in cases where there is a strong anisotropy energy coupling the magnetic moment and crystallographic axes of the particles. A second type of texture arises from the magnetic dipolar interactions between the particles, which can induce a 'spatial' texture. The paper considers the effects of both types of texture on the properties of ferrofluids.
\end{abstract}

\section{I - INTRODUCTION}

In the absence of a naturally occurring, strongly magnetisable fluid a ferrofluid is an interesting and useful substitute. A ferrofluid is a colloidal dispersion of ultrafine ferro-or-ferri-magnetic particles, with a magnetic susceptibility which is orders of magnitude larger than that of a paramagnetic liquid. In order to reduce the effects of the long-range magnetic dipolar interaction between the particles they are necessarily small (with diameters of the order of $10 \mathrm{~nm}$ ). . At this diameter thermal agitation is sufficient to ensure that the particles remain well dispersed. It is important also to counteract the attractive Van der Wals force which although short ranged is strong enough to cause irreversible aggregation of particles coming into contact. This is prevented by coating the particles with long chain surfactant molecules.

It is now possible to prepare ferrofluids of many different types using a variety of techniques. Of greatest practical significance are ferrofluids containing $\mathrm{Fe}_{3} \mathrm{O}_{4}$ (magnetite) particles because of their inherent chemical stability. These were originally prepared by ball milling /1/. More recently developed techniques /2/ utilise chemical techniques such as the precipitation of particles from mixed aqueous solutions of ferric and ferrous salts.

The magnetic properties of a ferrofluid are well demonstrated by its strong response to a magnetic field; this of course being the basis of most of their applications. The application of a magnetic field also has the effect of inducing a "texture' in the ferrofluid, which can be of two types. Firstly, a strong coupling between the magnetic moment and crystallographic axes of the particle results in a preferred particle orientation in a magnetised system (orientational texture). In addition, 
the dipolar interaction between particles can result in a 'spatial' texture ranging from short range order in the form of particle pairs to the highly anisotropic chain-like structures formed in strongly interacting systems.

The effects of texture are experimentally apparent in the anisotropic properties of ferrofluids such as the birefringence $/ 3,4 /$ and magnetodielectric effect $/ 5,6 /$ for example. This paper considers the effects of texture which are of some physical interest as well as presenting important possibilities for future applications. We also compare the two types of texture since it is recognised /5/ that both can explain existing data.

\section{II - OR IENTATIONAL TEXTURE AND THE MAGNETISATION PROCESS}

The particles of a ferrofluid consist of a single domain and behave effectively as a large magnetic moment $\mu=I_{s b} \nabla$ where $\nabla$ is the particle volume and $I_{s b}$ is the saturation magnetisation of the bulk material. The particles also possess an intrinsic magnetic anisotropy which could be magnetocrystalline in origin or (as is more often the case) arise from the non-spherical shape of the particles. In the solid state, the anisotropy has a profound bearing on the magnetic behaviour of the system, resulting in a significant coercivity and remanence. In a ferrofluid, however, the rotational freedom of the particles essentially decouples the particle anisotropy from the magnetisation process $/ 7,8, /$ resulting in a thermal equilibrium magnetisation independent of the anisotropy.

The reduced magnetisation $(\vec{I})$ of the system (relative to the saturation magnetisation $I_{s}$ ) is determined by the Langevin function:-

$$
\bar{I}=I(\beta)=\operatorname{coth} \beta-1 / \beta
$$

where $\beta=\mu \mathrm{H} / \mathrm{kT}$. In practice there will be a distribution of particle size, in which case the expression for the magnetisation must be modified as follows:-

$$
\bar{I}=\int_{0}^{\infty} F(v) d v \quad L(\beta),
$$

with $F(V) d V$ the distribution of volume fraction.

Equation (2) determines the magnetisation of a ferrofluid independently of the intrinsic anisotropy of the particles. It is important to note, however, that there are two distinct possible modes of magnetisation, which will depend on the energy product $K V$, where $K$ is the anisotropy energy density. When $K V$ is small it is possible for the magnetic moment to rotate independently of the crystallographic axes of the particle (Néel rotation). If $\mathrm{KV}$ is large the magnetic moment is more strongly coupled to the bulk of the particle which, as a consequence, must rotate in response to an applied magnetic field (Brownian rotation). Both mechanisms have been shown to occur in ferrofluids by $0^{\prime}$ Grady et al $/ 9 /$. clearly Brownian rotation results in particle alignment and is responsible for an 'orientational texture' of the system. By consideration of the dynamics of the magnetisation process, Shliomis /10/ has shown that Brownian rotation occurs above a critical volume $V$, given by $\mathrm{KV}$ - 4kT. However, the relaxation times are short (of the order of milliseconds), hence the system can normaliy be assumed to be in thermal equilibrium. In this case orientational texture will become important when the anisotropy energy becomes equal to the thermal energy. Thus we can define a critical volume $\nabla_{\text {such that }} \mathrm{KV}=\mathrm{kT}$, above which the coupling between the magnetic moment and the particle results in a significant orientational texture. As a first approximation it could be assumed that for $V>\nabla_{c}$ the coupling is perfect. However, this may seriously overestimate the degree of texture of the fluid, so we consider here the texture in a rather more general manner.

The energy $q^{f}$ a particle can be written as:$E_{p}=k \nabla \sin ^{2} \phi_{1}-\mu H \cos \theta_{1}$

with $\theta_{1}$ and $\phi_{1}$ the angles between the magnetic moment and the field and easy axis respectively. Equ. (3) is useful if it is required to calculate the probability of a particular $\phi_{1}$. In practice however, it is more useful to calculate thermally 
averaged quantities. In this case it is more convenient to use a coordinate system centered on the magnetic moment of a particle as shown in Figure 1 . In this coordinate system the energy of a particle is:$E_{p}=K V \sin ^{2} \omega-\mu H \cos \phi$.

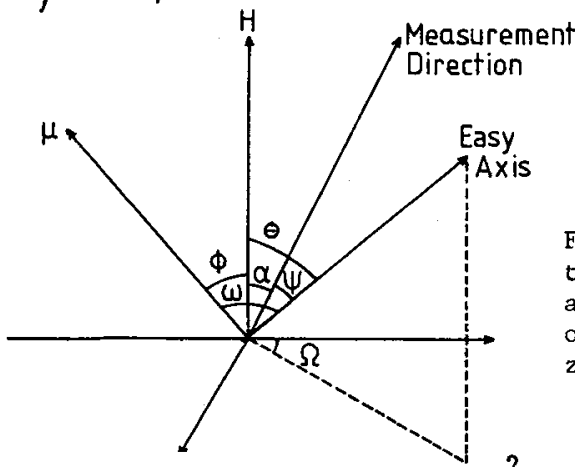

Fig. 1. Coordinate system used in the calculation. The equatorial angle of the easy axis in a coordinate system with $\mathrm{H}$ along the z-axis is $\xi$.

Often, the anisotropic properties depend on $\cos ^{2} \psi$ where $\psi$ is the angle between the particle easy axis and the measurement direction. This is the case, for example, in the observed birefringence and magnetodielectric anisotjopy of ferrofluids. Thus an important quantity is the thermal average denoted $\left\langle\cos ^{2} \psi\right\rangle$, for the system. Since the distribution must be symettric about $H$, it is more convenient to calculate (cos $\theta$ 〉 and geperalise this result using the fact that $(6 / 6 /)$$$
\left\langle\cos ^{2} \psi\right\rangle=\left\langle\cos ^{2} \theta\right\rangle \cos ^{2} \alpha+\frac{1}{2}\left(1-\left\langle\cos ^{2} \theta\right\rangle \sin ^{2} \alpha\right.
$$

where $\alpha$ is the angle between the field and the measurement direction. The average value of $\cos ^{2} \theta$ is given by:-

$$
\left\langle\cos ^{2} \theta\right\rangle=z^{-1} \int_{0}^{2 \pi} \int_{0}^{2 \pi} \int_{0}^{2 \pi} \sin \omega \sin \phi \cos ^{2} \theta \exp \left(-E_{p} / k T\right) d \omega d \phi d z
$$

Equation (6) has been evaluated previously/8/ giving (with a $=\mathrm{KV} / \mathrm{kT}$ and $\beta=\mu_{2} \mathrm{H} / \mathrm{kT}$ ).

$$
\begin{aligned}
& \left.\beta=\cos ^{2}\right\rangle=\left[1-\frac{2 L(\beta)}{\beta}\right] c(a)+\frac{L(\rho)}{\beta}[1-c(a)] \\
& \text { with } c(a)=\frac{\exp (a)}{a I(a)}-\frac{1}{2 a} \text {, and } I(a)=\int_{-1}^{1} \exp \left(a x^{2}\right) d x \text {. }
\end{aligned}
$$

We ngte that for strong coupling $(a \gg 1), c(a) \rightarrow 1$ and hence $\left\langle\cos ^{2} \theta>\sim 1-2 L(\beta) / \beta\right.$, which is the appropriate expression for perfect coupling. In addition as a $\rightarrow 0, c(a) \rightarrow 0$ and hence $\left\langle\cos ^{2} \theta\right\rangle=1 / 3$.

Thus the function $C(a)$ essentially determines the strength of the coupling between the magnetic moment and easy axis. In the strong coupling 1 imit it has been shown $/ 8 /$ that $c(a)$ is approximately given by :-

$$
c(a)=1-1 / a
$$

A numerical comparison shows that equ. (8) is valid for a> 1.5 .

\section{III - DIELECTRIC PROPERTIES OF A TEXTURED FERROFLUID}

We choose here to illustrate the effects of orientational texture by considering the field-induced dielectric anisotropy of a ferrofluid. This interesting 'magnetodielectric effect' is has received rather less attention than the well known field-induced birefringence, to which it must bear some. relation. The effect can be explained in terms of the orientation of ellipsoidal particles via the texturing mechanism outlined in section II. Assuming that the intrinsic magnetic and dielectric anisotropy arise from the particle elongation it has been shown /5,6/ that the relative permittivity of the ferrofluid, measured at an angle $\propto$ to the applied field is given by:$\epsilon_{\mathrm{r}}=1+\mathrm{f} \gamma+\mathrm{f} \delta \int_{0}^{\infty}\left\langle\cos ^{2} \psi\right\rangle \mathrm{F}(\mathrm{V}) \mathrm{d}$ 


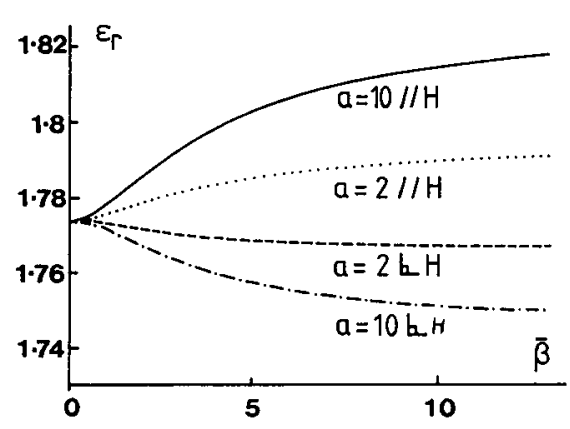

Fig 2. The variation of $\epsilon_{\text {r witil }} \bar{\beta}$ parallel and perpendicular to $i$ for $a=2$ and 10 .

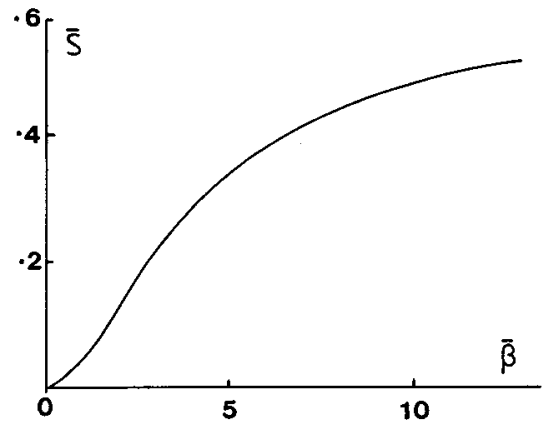

FIG 3. The order parameter $\bar{S}$ as a function of $\bar{\beta}$ for cobalt particles with $20 \%$ elongation

Here, $f$ is the volumetric packing fraction of the particles and the constants $\gamma$ and $f$ are given by:-

$$
\gamma=\left(\epsilon_{2}-1\right) !\left[1+\left(\epsilon_{2}-\epsilon_{1}\right) A_{2} / \epsilon_{1}\right]
$$

and $\delta=\left(\epsilon_{2}-1\right)\left[\left(1+\left(\epsilon_{2}-\epsilon_{1}\right) A_{1} / \epsilon_{1}\right)^{-1}-\left(1+\left(\epsilon_{2}-\epsilon_{1}\right) A_{2} / \epsilon_{1}\right)^{-1}\right]$

with $\epsilon_{2}$ and $\epsilon_{1}$ the relative permittivities of the particles and fluid respectively. In the case of metal particles, we simply set

$\epsilon_{2}=\infty$. The constants $A_{1}$ and $A_{2}$ are given in ref/5/, and for small particle elongation (e) reduce to:

$A_{1}=e^{2}\left(1 / 3+\left(1-e^{2}\right) / 5\right), A_{2}=\left(1-A_{1}\right) / 2$

Thus $\epsilon_{r}$ measured at some angle $\alpha$ to the applied field is given by:

$\epsilon_{\mathrm{r}}=1+f \gamma+f \delta \int_{0}^{\infty} \mathrm{F}(V)\left\{\left\langle\cos ^{2} \theta\right\rangle-\sin ^{2} \alpha\left[\frac{3}{2}\left\langle\cos ^{2} \theta\right\rangle-\frac{1}{2}\right]\right\} \mathrm{dV}$

Fig 2 shows the variation of $\epsilon_{\text {r }}$ parallel and perpendicular to $H$ with the parameter $\bar{\beta}=I_{s b} V_{m} H / k T$ for magnetite particles with $20 \%$ elongation in a carrier of relative permittivity $\epsilon_{1}=2$, for various values of $a=K V_{m} / k T$ which determines the strength of the coupling. This shows that ${ }_{a}$ maximum change in $\epsilon_{r}$ of a few percent is to be expected. Differention of $(10)$ shows that

$$
\frac{d}{d \alpha} \epsilon_{r}=-f \delta \sin 2 \alpha \int_{0}^{\infty} F(V)\left[\frac{3}{2}\langle\cos \theta\rangle-\frac{1}{2}\right] d V
$$

The quantity d $\epsilon_{r} / d \propto$ has a characteristic variation with sin $2 \alpha$. Equ (11) also involves the volume weighted average of a liquid-crystal like order parameter $(3<\cos \theta>-1) / 2$. This quantity, denoted $\overline{\mathrm{s}}$ is representative of the orientational texture for any given field. The variation of $S$ with field for a dispersion of cobalt particles with $a=5$ and an elongation of $20 \%$ is shown in fig 3 . The carrier fluid has $\epsilon_{2}=2$.

It is possible to derive expressions for the asymptotic behaviour of equ (10). In the limit of small fields, $\beta<1$ and using the low field apprgximation to the Langevin function,

$\left\langle\cos ^{2} \theta\right\rangle=\frac{1}{3}+\frac{2}{45} \beta^{2}(3 c(a)-1)$.

Similarly in the limit of large fields, $\beta \gg>1$ and hence

$$
\left\langle\cos ^{2} \theta\right\rangle=1-\frac{1}{\beta}(3 \mathrm{c}(\mathrm{a})-1)
$$

These expressions lead to the results (for $\alpha=0$ ):

$$
\epsilon_{\mathrm{r}}=1+f \gamma+\frac{2 f \delta \mathrm{H}^{2} \mathrm{~T}^{2}}{45 k^{2} T^{2}} \int_{0}^{\infty} \mathrm{V}^{2}(3 \mathrm{c}(\mathrm{a})-1) \mathrm{F}(\mathrm{V}) \mathrm{dV}
$$


for $\beta<1$, and for $\beta>1$,
$\epsilon_{\mathrm{r}}=1+\mathrm{f} \gamma+\mathrm{f} \delta\left\{1-\frac{\mathrm{kT}}{\mathrm{I} \mathrm{g}} \int_{0}^{\infty} \mathrm{v}^{-1}(3 \mathrm{c}(\mathrm{a})-1) \mathrm{F}(\mathrm{V}) \mathrm{dV}\right\}$

Thus we expect a linear dependence of $\varepsilon_{r}$ in the low field region and in high fields a $1 / \mathrm{H}$ extrapolation to a maximum value $\epsilon_{\mathrm{r}}=1+\mathrm{f} \gamma+\mathrm{f} \delta$. The predictions of equ (14) and (15) have Eeen confirmed experimentally by measurements made in this laboratory/11/.

The predictions of the theory are broadly in agreement with published data on the magnetodielectric effect. Such measurements can clearly provide useful information on the orientational texture of a ferrofluid. Further work is necessary in order to investigate the frequency dependence observed by Cotae/12. Measurements of the parameter $\bar{s}$ would also give $a$ useful guide to the degree of orientational texture in ferrofluids.

We have illustrated the effects of texture by consideration of the magnetodielectric effect. The theory can, however, easily be applied to any field-induced anisotropy caused by orientational texture, for example the torque on a ferrofluid solidified in a large applied field/8/ in which the orientational texture is frozen in. The situation is complicated, however, by the possibility of a spatial texture arising from interparticle interactions which is often invoked as an explanation for the field induced anisotropic behaviour of ferrofluids. For example Cotae/12/ has explained the magnetodielectric effect in terms of interactions. Spatial texture is considered in the following section.

IV - SPATIAL TEXTURE IN FERROFLUIDS - MONTE CARLO CALCULATIONS

In an ultra-stable ferrofluid the requirement of small particle diameter is paramount so that the attractive forces between particles are minimised. Because of the long-range nature of the dipolar interaction, however, this does have a bearing on the properties of systems with a high degree of colloidal stability. One example of this is the observation/13/of a Curie-Weiss like variation of the initial susceptibility with temperature; an effect which can be explained in terms of interparticle interactions. An important consequence of interparticle interactions is a degree of spatial ordering of the particles, which can be considered as a 'spatial texture", since it can give rise to anisotropic properties similar to those arising from the orientational texture discussed previously.

Depending on the system, the consequences of interactions range from short liquid-1ike order to the highly anisotropic chain configurations observed in large magnetic fields. Analytical theories include that of Jordan/14/, lattice gas models/15/ and a calculation by Hayter and Pynn/16/ of the pair correlation function of a saturated ferofluid. Here we shall consider a computational technique based on the MonteCarlo method, which has the advantage of providing 'exact' results (within the statistical error) for a given model system.

The Monte-Carlo method entails the generation of a Markov chain of points in phase space in which the transition probability between neighbouring points is taken to be exp(- $\Delta E / k T)$ where $\Delta E$ is the energy difference. In practice a representative cell of $N(\sim 1000)$ particles is set up, and each particle in turn given a random displacement in its coordinates (including the associated magnetic moment). The energy difference ( $\triangle E$ ) between the two positions is calculated and the trial move accepted with a probability exp ( $-\Delta E / k T)$. Periodic boundary conditions are used to extend the cell, i.e. if a particle leaves the cell it is assumed to re-enter on the opposite 

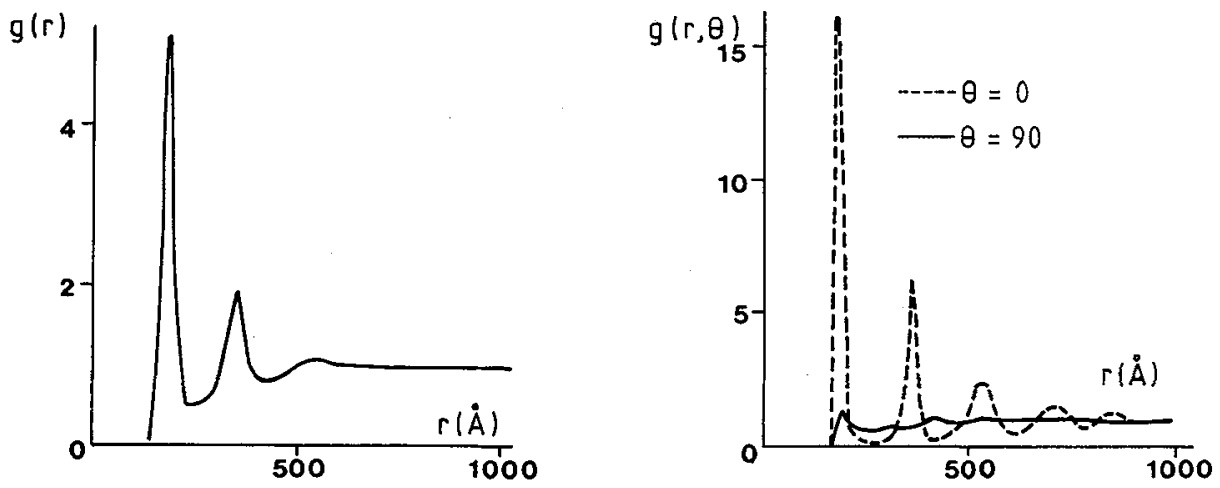

FIG 4. $g(r)$ for $150 \AA ̊$ diameter cobalt particles FIG 5. $g(r)$ for $150 \AA$ diameter cobalt particles in zero field. In this case $g(r)$ is isotropic. in a field of $10 \mathrm{k} 0 \mathrm{e}$. The field-induced anisotropy can clearly be seen.

8ide. After many Monte-Carlo 'moves' per particle the system can be shown $/ 17 /$ to evolve into a thermal equilibrium configuration, characterised by a Boltzmann distribution of the energy.

For the case of strongly interacting particles, the Monte-Carlo aproach has shown /18/ a spatial texture dependent on the applied field. The spatial texture can be assessed by calculating the pair distribution function $g$ (r) defined as the average number density at a distance $\underline{r}$ from a central particle, normalised with respect to the average number density of the fluid. Figures 4 and 5 show $g$ (r) for $150 \AA$ diameter cobalt particles in applied fields of zero and $10 \mathrm{k} 0 \mathrm{e}$ respectively. $g(\underline{r})$ is given as $g(r, \theta)$ where $\theta$ is the angle the line joining the particle centres makes with the applied field. The zero field configuration shows spatial order, having a pronounced nearest neighbour peak. the anisotropy in the spatial texture in a field of 10 koe can clearly be seen in fig 5, where $g(\underline{r})$ has a large nearest neighbour peak for $\theta=0$, with little correlation apparent in a direction perpendicular to $H$.

It is reasonable to expect that the spatial texture could contribute to the anisotropic properties of ferrofluids. We shall again illustrate this with reference to the magnetodielectric effect. Consider a ferrofluid containing particles of polarisability $\alpha_{e}$. The particles are assumed to be perfectly spherical, so that orientational texture can be neglected. In this situation the electrostatic interactions between the particles will have a bearing on the dielectric response of the ferrofluid. Assume for simplicity that parallel electric and magnetic fields are applied to a ferrofluid, and consider the electrostatic interactions between a pair.of particles which make an angle $\theta$ to the applied field. The component of the mutual interaction field parallel to the applied field (E) is

$$
E_{i}=\frac{p}{4 \pi \epsilon_{0} r^{3}}\left(3 \cos ^{2} \theta-1\right)
$$

where $p$ is the electric dipole moment and $r$ is the particle separation. The dipole moment is $p=\alpha_{e}\left(E+E_{i}\right)$, and since the electric susceptibility is $x_{e}=n_{p} / \epsilon_{0} E$ (with ${ }^{2}$ the number of particles/unit volume) we have that

$$
\mathcal{X}_{\mathrm{e}}=\frac{\mathrm{n} \alpha_{e}}{\epsilon_{0}}\left[1-\frac{\alpha_{e}}{4 \pi \epsilon_{0} r^{2}}\left(3 \cos ^{2} \theta-1\right)\right]^{-1}
$$


Further, the relative permittivity is $\epsilon_{r}=1+\epsilon_{0} X_{e}$, and since the electric interaction is relatively weak the second term in equ ( 16 ) will be smal1. Thus to a first approximation, for a system with a distribution of $\theta$,

$$
\epsilon_{\mathrm{r}}=1+\frac{\mathrm{n} \alpha_{e}}{\epsilon_{0}}+\frac{2 \mathrm{n} \alpha_{e}^{2}}{4 \pi \epsilon_{0}^{2} \mathrm{r}^{3}} \mathrm{~s}
$$

where $S=\left\langle\frac{3}{2} \cos ^{2} \theta-1 / 2\right\rangle$ is the orientational order parameter.

The magnitude of the effect can be assessed by substituting the value of $x_{e}$ which can be determined from the work of colteu/I9/ as

$$
\alpha_{e}=v \epsilon_{0}\left(\epsilon_{2}-1\right)\left\{1+\left(\epsilon_{2}-\epsilon_{1}\right) / 3 \epsilon_{1}\right\}^{-1}
$$

This gives a texture dependent contribution to the relative permittivity as

$$
\Delta \epsilon_{r} \quad=\frac{f V\left(\epsilon_{2}-i\right)^{2} S}{2 \pi r^{3}\left(1+\left(\epsilon_{2}-\epsilon_{1}\right) / 3 \epsilon_{1}\right)^{2}}
$$

where $f=n V$ is the volumetric packing fraction. For reasonable values of $\epsilon_{1}$ and $\epsilon_{2}$, the maximum value of $\Delta \epsilon_{r}$ (for particles in contact) is of the order of $f$, hence the pair interaction mechanism is of the correct magnitude to explain the experimental data, which show a maximum value of $\Delta \epsilon_{r}$ of a few percent. This serves to show that the pair orientation mechanism can explain the observed dielectric anisotropy in ferrofluids. Further work is necessary, however, in order to determine the functional variation of $E_{r}$ with $H$ and to effect a comparison with the pair orientation mechanism.

\section{$\nabla$ - DISCUSSIOH}

We have considered two types of texture which can occur in ferrofluids. Orientational texture is an intrinsic property of the particles and will arise to a degree in all ferrofluids. Spatial texture is a co-operative phenomenon and will occur to an extent dependent on the interaction strength between the particles. of the two the latter is the least well understood and is worthy of further investigation. There are cases where one type of texture is dominant, for example, the anisotropic properties of water based fluids are likely to be primarily due to the long chains which have been observed/20/ on the application of large applied fields to those systems.

In more weakly interacting systems it is likely that spatial and orientational texture will both contribute to the anisotropic properties of ferrofluids. Here the spatial texture is not as pronounced as in the case of strong interactions. It has been shown, however, using the Monte-carlo method/21/ that short ranged order involving particle pairs exists. Additional analytical calculations/22/, based on the evaluation of an approximate partition function have shown that the axes of the pairs can be oriented by an applied field, resulting in a field dependent spatial texture. This pair orientation mechanism will exist even in ultra-stable ferrofluids, since it requires only short range order which will not be detrimental to the colloidal stability of the ferrofluid.

The effect of orientational texture and weak spatial texture may be difficult to distinguish. For example the calculations of chantrell et al $122 /$ show that $\underset{3}{ }$ h 2 dimensions, correct to first order in the constant $\lambda=2 \mu^{2} / \mathrm{kTr}^{3}$ (with $\mathrm{r}$ the average particle separation). 
$\left\langle\cos ^{2} \theta\right\rangle=\left[I_{0}^{2}(\beta)+5 \lambda_{1}^{2}(\beta) / 8\right] /\left[2 I_{0}^{2}(\beta)+\lambda I_{1}^{2}(\beta) / 2\right]$

where $I_{n}(x)=(2 \pi)^{-1} \int_{0}^{2 \pi} \cos ^{n} \theta \exp (x \cos \theta) d \theta$

Equ (19) has a variation with field which is rather similar to that expected for orientational texture. In $2-D$ the equivalent order parameter in equ (18) is $s=2\left\langle\cos ^{2} \theta\right\rangle \overline{-} 1$. In $s m a l 1$ fields it has been shown $/ 22 /$ that $\left\langle\cos ^{2} \theta\right\rangle=1 / 2+3 \beta^{2} / 40$, hence $s \alpha \mathrm{H}^{2}$ and consequentiy the relative permittivity $\epsilon_{\text {is dependent on }}{ }^{2}$ as is the case for orientational texture. Similarly, using the high field approximation to $I_{1}(\beta)$ and $I_{2}(\beta)$ we have that $S=9 \lambda(1-3 /(4 \beta))$. In this limit therefore we expect from (17) a $1 /$ H extrapolation to a saturation value of $\epsilon_{r}$; again the same behaviour as for orientational texture. Thus in these asymptotic limits there is no distinction between the effects of orientational and spatial texture.

One possibility to distinguish between orientational and spatial texture is in the variation of the saturation value of $\epsilon_{r}$ with the volumetric packing fraction f. In the case of orientational texture we expect a simple linear variation of the saturation value with f. In the case of spatial texture, however, we expect a more complex variation. Here $\Delta E_{r} \propto f 5 / r^{3}$ and since both $S$ and $r$ are likely to depend on $f$ it is unitkely that the maximum value of $\epsilon_{r}$ will be linearly dependent on $f$. Thus the variation of $\epsilon_{r}$ with concentration would be an interesting experimental study. Further theoretical work is also necessary in order to determine the precise variation of $\epsilon_{r}$ with f for the spatial texture mechanism. This could be caried out using the Monte-Carlo model outlined previously.

\section{REFER ENCES}

1. R.E. Rosensweig, J.W. Nestor and R.S. Timmins AIChE - IChem E symp Ser 5 (1965) 104

2. S.E. Khalafalla and G.W. Reimers IEEETrans Mag MAG-16(1980) 178

3. J.P. Llewel1yn, J. Phys D 16 (1983) 95

4. M.M. Maiorov and 0. Tsebers Col1 J 39 (1977) 954

5. A.J. Mailfert and B. Nahounou, IEEE Trans. Mag. MAG-16, (1980) 254

6. R.W. Chantrel1, J. Magn.Magn. Mater 45, (1984) 100

7. D.A. Krueger, J. App 1. Phys. 50, (1979) 8169

8. R.W. Chantrei1, S.R. Hoon and B.K. Tanner J. Magn.Magn. Mater 38 (1983)

9. R. O'Grady, J. Popplewel1 and S.W. Charles J. Magn. Magn. Mater 39 , (1983) 56

10. M.I. Shiiomis Sov Phys USP 17 (1974) 153

11. P.R. Bissel1, Private communication

12. C. Cotae, J. Magn. Magn. Mater 39 (1983) 85

13. K. 0’Grady, A. Bradbury, S.W. Charles, S. Menear, J. Popplewell and R.W. Chantrel1 J.Magn.Magn. Mater 31-34 (1983) 958

14. P.C. Jordan Mol Phys. 38 (1979) 769

15. M. Ausloos, P. Clippe, J.M. Kowalski, J. Pekalska and A. Pekalski J. Magn. Magn. Matex 39 (1983) 21

16. J.B. Hayter and R. Pynn Phys Rev Lett 49 (1982) 1103

17. K. Binder (ed) Monte Carlo methods in statistical physics, Springer-Verlag (1979)

18. R.W. Chantrel1, A. Bradbury, J. Popplewel1 and S.W. Charles J. App 1. Phys 53 (1982) 2742

19. A. Colteu J. Magn.Magn. Matex 39 (1983) 88.

20. A. Martinet in 'Thermomechanics of magnetic fluids', B. Berkovsky (ed), Hemisphere (1978)

21. S. Menear, A. Bradbury and R.W. Chantrel1 J. Magn.Magn. Mater $4 \underline{3}$ (1984) 166

22. R.W. Chantre11, A. Bradbury and S. Menear.J.App1.Phys (in Press) 